$54^{\text {ème }}$ Congrès de la SFMBCB, 03012 (2011)

DOI: $10.1051 / \mathrm{sfmbcb} / 20115403012$

(C) Owned by the authors, published by EDP Sciences, 2011

\title{
A propos de la prise en charge de 2 cas d'ostéite chronique survenue sur des foyers de dysplasie ostéo-cémentaire
}

\author{
Fernet $\mathrm{J}^{1}$, L'Homme $\mathrm{A}^{1,2}$, Cousty $\mathrm{S}^{1,2}$, Courtois $\mathrm{B}^{1,2}$, Duran $\mathrm{D}^{1,2}$ \\ ${ }^{1}$ UFR d'Odontologie, Université Paul Sabatier, Toulouse III, France \\ ${ }^{2}$ Service d'Odontologie hospitalière, CHU, Toulouse, France \\ jessica.fernet@wanadoo.fr
}

La dysplasie ostéo-cémentaire constitue la lésion fibro-osseuse des maxillaires la plus fréquente. Il s'agit d'une lésion bénigne au sein de laquelle l'architecture osseuse est progressivement remplacée par du tissu fibreux avec une composante minérale, cémentaire variable selon le stade de maturation. A un stade avancé, il en résulte une zone peu vascularisée et pauvre en éléments figurés du sang, particulièrement sensible aux infections (Waldron 2002). La dysplasie ostéo-cémentaire se présente le plus souvent comme une lésion asymptomatique, unique ou multiple, plus ou moins bien limitée. Elle concerne plus fréquemment les populations d'Afrique subsaharienne ou d'Afrique du Nord (64\%), et touche surtout les femmes (89\%). A l'origine de cette lésion, on suspecte l'implication du ligament alvéolo-dentaire, en raison de sa composition et de sa localisation dans l'os alvéolaire.

L'aspect radiologique varie selon le stade d'évolution : lacune radio-claire à un stade précoce, puis image mixte, et radio-opaque à un stade avancé. Les manifestations cliniques, peu fréquentes, sont dominées par les déformations osseuses, principalement dans les formes florides, et la douleur qui fait généralement suite à l'exposition buccale du foyer de dysplasie (Cory 2008). La distinction entre dysplasie ostéo-cémentaire et les autres lésions osseuses permet d'adapter l'attitude thérapeutique puisque l'abstention et la surveillance sont de rigueur pour les lésions de dysplasie ostéo-cémentaire asymptomatiques.

Nous rapportons deux cas de dysplasie ostéo-cémentaire devenues symptomatiques après mise en contact avec la cavité buccale. Le premier concerne une patiente de 81 ans, adressée pour des douleurs mandibulaires persistantes depuis l'avulsion de la 36 quinze mois auparavant. Le site d'avulsion montrait une alvéole béante, exposant l'os alvéolaire jusqu'au contact de la dent 37 . Sur la radiographie panoramique, on notait une image d'ostéo-condensation nuageuse sur le site exposé, et des images radio-claires et mixtes dans la région apicale des prémolaires et des molaires mandibulaires, permettant de porter le diagnostic de dysplasie ostéo-cémentaire. L'examen tomodensitométrique réalisé pour évaluer les rapports de la dysplasie avec le nerf alvéolaire inférieur montrait une continuité de la lésion avec les corticales vestibulaire et linguale. Le foyer d'ostéite, très peu hémorragique, a été éliminé par fraisage osseux jusqu'à obtention d'un saignement osseux. La 37, totalement ankylosée dans l'os dysplasique, a du être avulsée dans le même temps. Le second cas concerne une patiente de 28 ans, adressée pour des douleurs mandibulaires irradiantes, persistantes après une résection apicale réalisée sur la 36, plus d'un an auparavant. Le diagnostic d'ostéite mandibulaire sur foyer de dysplasie ostéo-cémentaire a été porté sur la radiographie panoramique qui montrait des foyers de dysplasie multiples dans les régions molaires mandibulaires. La 36 a été avulsée et le foyer d'ostéite, peu hémorragique, cureté jusqu'à obtention d'un plan osseux capable de générer un caillot.

Dans les deux cas, le site opératoire a été refermé par des sutures et isolé du milieu buccal par l'application d'une colle de fibrine (Tissucol $\left.{ }^{\circledR}\right)$. 